\title{
Hybrid cervical disc arthroplasty
}

\author{
Tsung-Hsi Tu, MD, ${ }^{1-3}$ Jau-Ching Wu, MD, PhD, ${ }^{1,2}$ Henrich Cheng, MD, PhD, ${ }^{1,2}$ \\ and Praveen V. Mummaneni, MD 4
}

\begin{abstract}
1Department of Neurosurgery, Neurological Institute, Taipei Veterans General Hospital; ${ }^{2}$ School of Medicine, National Yang-Ming University; ${ }^{3}$ Taiwan International Graduate Program in Molecular Medicine, National Yang-Ming University and Academia Sinica, Taipei, Taiwan; and 4Department of Neurological Surgery, University of California, San Francisco, California
\end{abstract}

For patients with multilevel cervical stenosis at nonadjacent segments, one of the traditional approaches has included a multilevel fusion of the abnormal segments as well as the intervening normal segment. In this video we demonstrate an alternative treatment plan with tailored use of a combination of anterior cervical discectomy and fusion (ACDF) and cervical disc arthroplasty (CDA) with an intervening skipped level.

The authors present the case of a 72-year-old woman with myeloradiculopathy and a large disc herniation with facet joint degeneration at C3-4 and bulging disc at C5-6. After nonoperative treatment failed, she underwent a single-level ACDF at C3-4 and single-level arthroplasty at C5-6, which successfully relieved her symptoms. No intervention was performed at the normal intervening C4-5 segment. By using ACDF combined with arthroplasty, the authors have avoided a 3 -level fusion for this patient and maintained the range of motion of 2 disc levels.

The video can be found here: https://youtu.be/OrxcPUBvqLk.

KEYWORDS anterior cervical discectomy and fusion; ACDF; arthroplasty; spondylosis; video 